DOI: 10.12731/2227-930X-2021-11-1-87-98

УДК 004.93

\title{
РАЗРАБОТКА СИСТЕМЫ ДЛЯ АНАЛИЗА И РАЗГРУЗКИ ДОРОЖНОГО ТРАФИКА С ПРИМЕНЕНИЕМ ИСКУССТВЕННОГО ИНТЕЛЕКТА
}

\author{
Ахметов Л.М., Биков Д.И., Хамидуллин М.Р.
}

С каждым годом темпы роста автомобилей в России продолжают расти, что крайне усложняет организацию дорожного трафика. Для решения этой проблемы необходимы инноващии в автомобильной отрасли, такие как умныле светофоры, для более эффективного регулирования движения транспорта на дорогах общего пользования.

Цель - разработка системы для анализа и разгрузки дорожного трафика для улучшения ситуации на перекрестках, автоматизация путём внедрения искусственного интеллекта, сборка рабочего макета светофора на базе Arduino.

Метод или методология проведения работы: в статье рассмотрен проект по анализу, отслежсиванию и разгрузке транспорта на перекрестках.

Результаты: собран макет светофора на базе микроконтроллера Arduino UNO и разработана нейронная сеть для анализа и разгрузки дорожного трафика в реальном времени на языке программирования Python.

Область применения результатов: полученные результаты и̧елесообразно применять на наиболее перегруженных участках дорог.

Ключевые слова: Светофор, Дорожное движение; Arduіпо; Автоматизация; Анализ; Нейронная сеть; Python

\section{DEVELOPMENT OF A SYSTEM \\ FOR ANALYZING AND UNLOADING ROAD TRAFFIC USING ARTIFICIAL INTELLIGENCE Akhmetov L.M., Bikov D.I., Khamidullin M.R.}

Every year the growth rate of cars in Russia will continue to grow, which will complicate the organization of road traffic. Therefore, in- 
novations in the automotive industry, such as smart traffic lights, are needed to regulate traffic more efficiently.

Purpose - development of a system for analyzing and unloading road traffic to improve the situation at intersections, automating road traffic by introducing artificial intelligence, assembling a working model of a traffic light based on Arduino.

Method or methodology of work: the article considers a project to analyze and track and unload vehicles at an intersection.

Results: a mock-up of a traffic light based on an Arduino UNO microcontroller was assembled and a neural network was developed for analyzing and unloading road traffic in real time in Python.

Scope of the results: the results obtained should be applied to the most congested road sections.

Keywords: Traffic light, Road traffic; Arduino; Automation; Analysis; Neural network; Python

\section{Введение}

В данной статье рассматривается одно из возможных решений проблемы низкого уровня алгоритма регулировки светофоров на перекрестках. Подходы, изложенные в данной работе, описывают механизм оптимизации перекрестков с применением искусственного интеллекта и алгоритма разгрузки. Данную систему целесообразно применять на наиболее перегруженных участках дорог во многих городах России [11].

Разработка системы анализа и разгрузки дорожного трафика направлена на отслеживание количества транспорта в реальном времени и взаимодействия со светофором с использования компьютера [10].

Принцип функционирования системы заключается в том, что через камеры предобученная нейронная сеть распознает транспортные средства и анализирует их. В зависимости от количества транспорта на каждой стороне дороги, светофор меняет цвет [6]. Например, если в одной стороне дороги находится 6 машин, а в другой 2, то таймер светофора выставится на 20 секунд. Длительность зависит от соотношения объектов на дороге [9]. 


\section{Материалы и методы}

Макет светофора собран на Arduino UNO - это плата микроконтроллера на базе ATmega328P [1]. Основные характеристики Arduino UNO, представлены в таблице 1.

Таблицุа 1.

Основные характеристики платформы

\begin{tabular}{|c|c|}
\hline Микропроцессор & ATmega328 \\
\hline Рабочее напряжение & 5 В \\
\hline Входное напряжение(рекомендуемое) & $7-12$ В \\
\hline $\begin{array}{c}\text { Входное напряжение } \\
\text { (приемлемое) }\end{array}$ & $6-20$ В \\
\hline Цифровые Входы/Выходы & 14 \\
\hline Аналоговые входы & 6 \\
\hline Постоянный ток через вход/выход & $40 \mathrm{MA}$ \\
\hline Постоянный ток для вывода 3.3В & $50 \mathrm{MA}$ \\
\hline Флеш-память & 32 Кб \\
\hline ОЗУ & 2 Кб \\
\hline ЕЕРRОМ & 1 Кб \\
\hline Тактовая частота & 16 Мгц \\
\hline
\end{tabular}

Основной принцип работы устройства заключается в передаче видеосигнала на компьютер через 2 веб-камеры в нейронную сеть, определяется количество транспорта на дороге, далее происходит визуализация ситуации на перекрестке в приложении и через СОМ-порт данная информация передаются на плату Arduino и включается светофор [2].

Иллюстрация макета светофора приведена ниже на рисунках 1, 2. Макет состоит из:

1) 6 светодиодов

2) 6 резисторов на $220 \mathrm{OM}$

Для начала работы программы необходимо 2 веб-камеры, компьютер и подключение платы Arduino в СОМ-порт, последующим включением самой программы [3]. 


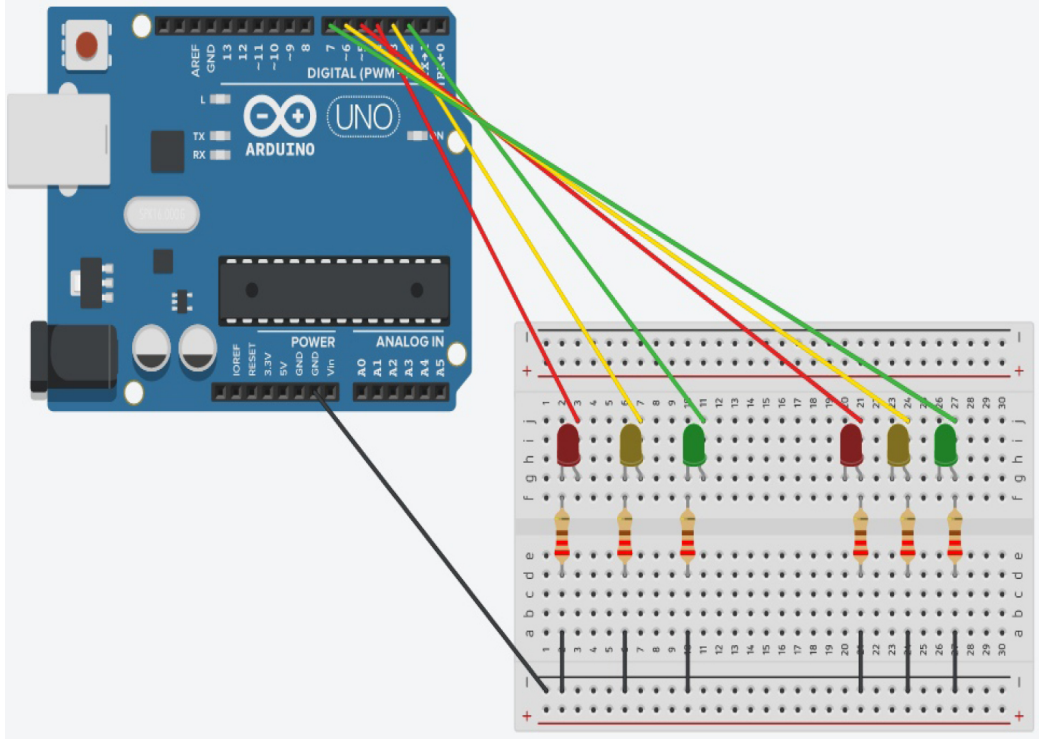

Рис. 1. Схема подключения макета светофора

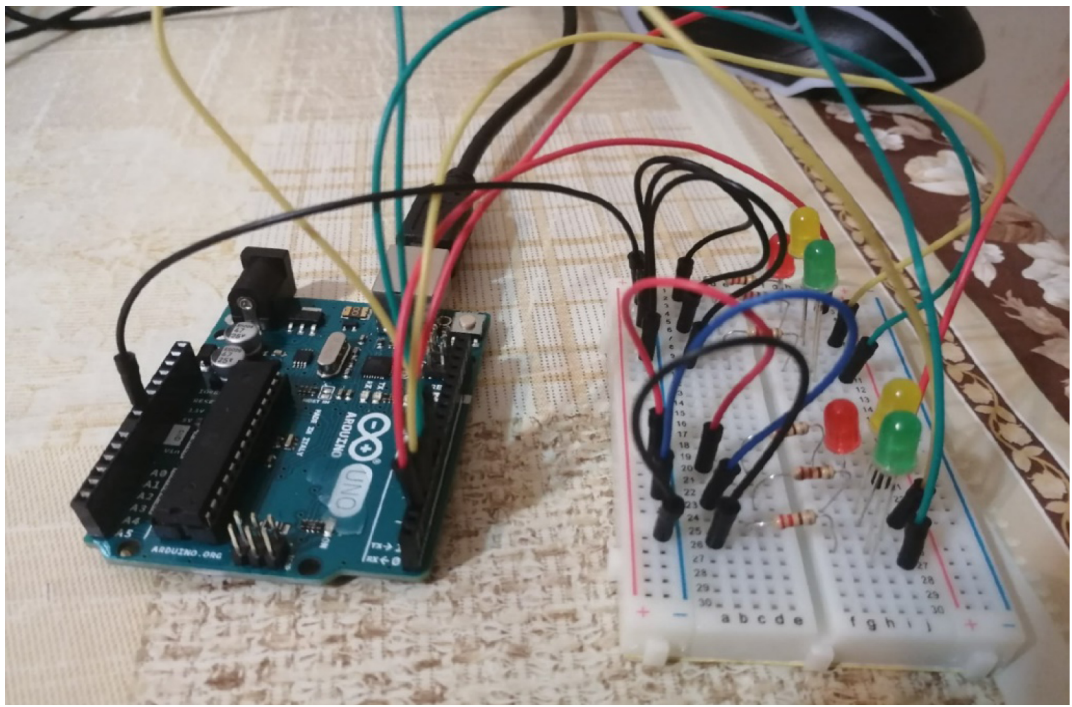

Рис. 2. Иллюстрация макета вид сбоку 


\section{Механизм работы светофора:}

- Красный;

- Красный + желтый;

- Зеленый;

- Желтый;

- Повторение.

Программная реализация светофора в среде Arduino IDE (рис 3).

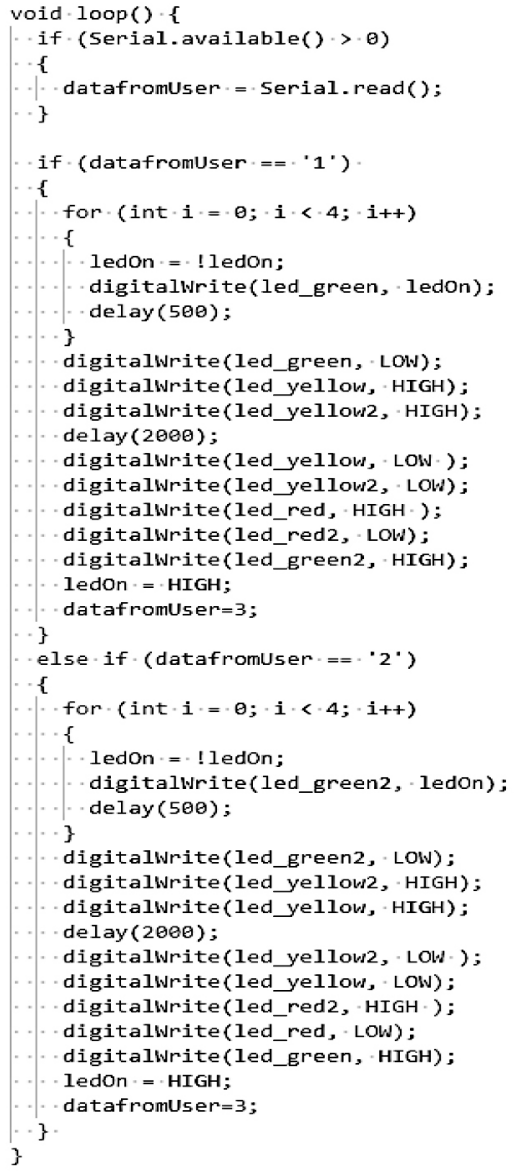

Рис. 3. Пример скетча Arduino работы светофора 
Для создания десктопного приложения использовались следующие библиотеки Python [12]:

OpenCV - библиотека, ориентированная на приложения реального времени, используется для видеоанализа с веб-камеры количества машин, находящихся на дороге [4].

ImageAI - библиотека для прогнозирования изображений, обнаружения и отслеживания видеообъектов.

PyQt5 - библиотека для разработки пользовательского интерфейса приложения.

PySerial - библиотека для работы с COM-портами, используется для связи микроконтроллера Arduino UNO с Python [13].

\section{Результаты}

Разберем основные достоинства и интерфейс программы:

1) Возможности

2) Интуитивность

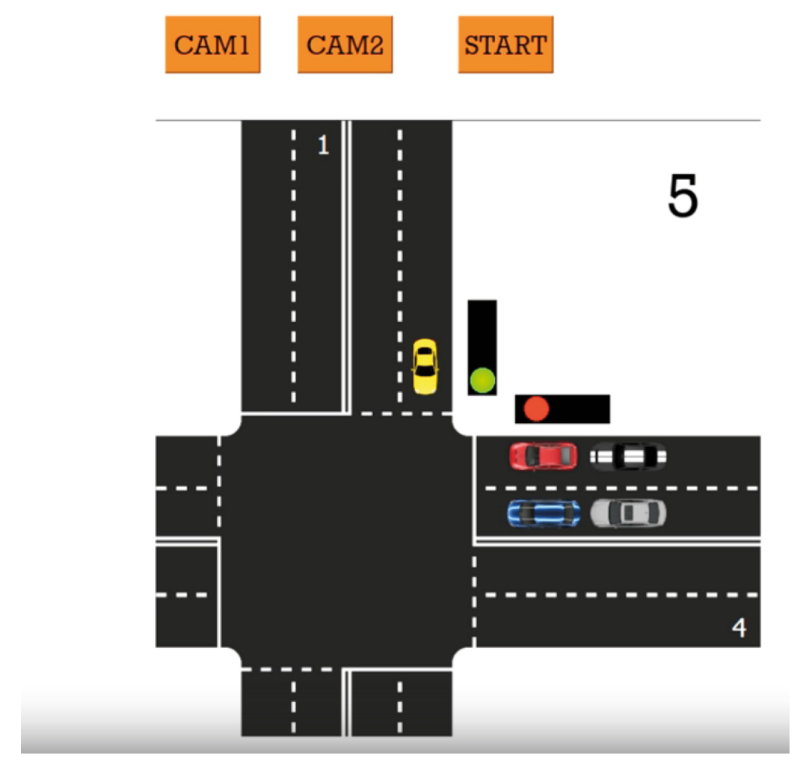

Рис. 4. Интерфейс 


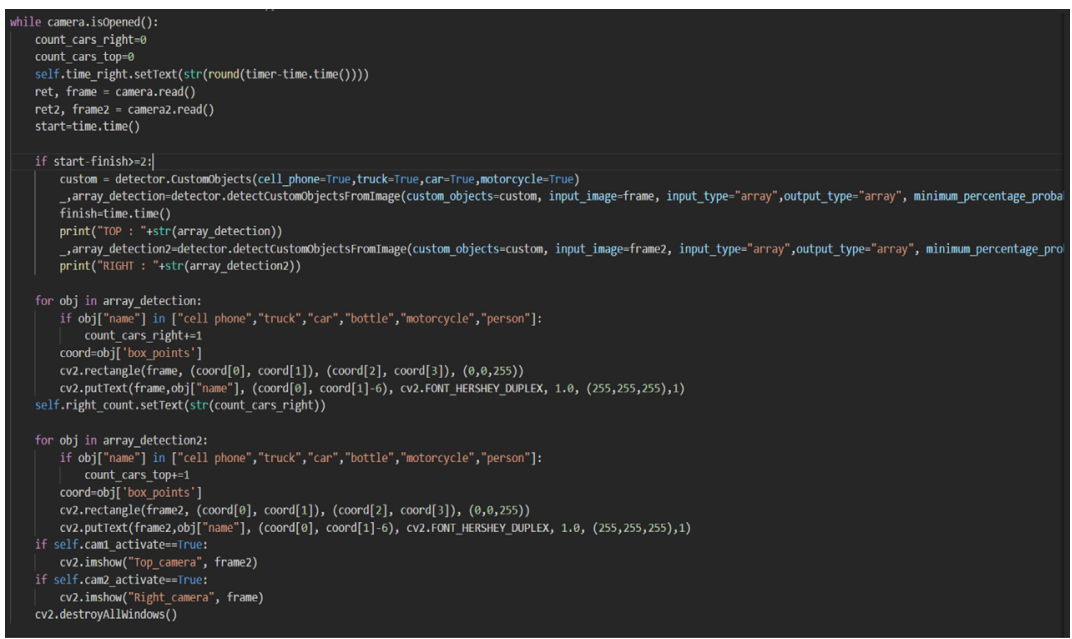

Рис. 5. Код для обнаружения автомобилей на дороге

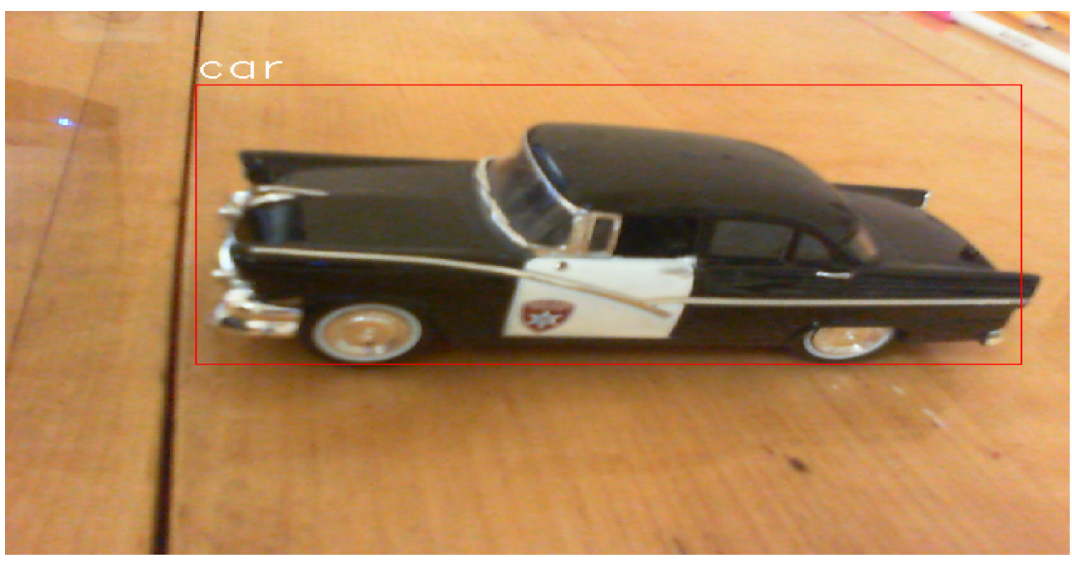

Рис. 6. Пример демонстрации объекта

Распознавание автомобилей на дороге происходит при помощи инструментов библиотеки ImageAI и заранее обученной модели «yolo.h5» [5]. Программа в каждой итерации цикла while проверяет изображение, полученное с веб-камер при помощи библиотеки OpenCV и сравнивает полученные объекты с заранее обученными 
моделями yolo [14]. Если модели совпадают, программа сохраняет позиции х и у распознанного объекта и рисует вокруг него прямоугольник (Рис 5).

После всех манипуляций скрипта, обработанное изображение демонстрируется на экран при помощи функции imshow (рис 6).

\section{Выводы}

Был собран макет светофора на базе микроконтроллера Arduino UNO сопряженный с компьютером, способный имитировать и разгружать дорожный трафик, решать проблему низкого уровня алгоритма регулировки светофоров на перекрестках, позволяющий усовершенствовать движение транспортных средств на перегруженных участках дороги [7].

Разработана и настроена нейронная сеть на языке Руthon способная отслеживать видеопоток и анализировать количество транспортных средств и разгружать дорожный трафик [8]. Учитывая вышесказанное, можно сделать вывод, что разработка с учетом модернизаций, способна удовлетворять всем необходимым нуждам и способна значительно улучшить и автоматизировать ситуацию на дорогах.

\section{Список литературы}

1. Акмаров П.Б. Кодирование и защита информации : учебное пособие. Ижевск: Ижевская ГСХА, 2016. 136 c. https://e.lanbook.com/ book/133975 (дата обращения: 28.02.2020).

2. Богачёв К.Ю. Основы параллельного программирования. 3-е изд. (эл.). Москва: БИНОМ. Лаборатория знаний, 2015. 345 с. https://ibooks.ru/reading.php?productid=350082 (дата обращения: 28.02.2020).

3. Гарибов А.И. Основы разработки приложений для мобильных устройств на платформе Windows Phone : учебное пособие. 2-е изд. Москва: ИНТУИТ, 2016. 459 c. https://e.lanbook.com/book/100344 (дата обращения: 28.02.2020).

4. Иванова Г.С. Технология программирования : учебник. М.: КНОPУC, 2011. $336 \mathrm{c}$. 
5. Подбельский В.В. Язык декларативного программирования XAML. Москва: ДМК Пресс, 2018. 336 c. https://e.lanbook.com/ book/111428 (дата обращения: 28.02.2020).

6. Пономаренко В.И., Караваев А.С. Использование платформы Arduino в измерениях и физическом эксперименте // Известия Вузов. ПНД. 2014. Т. 22, №4. С. 77-90.

7. Рудинский И.Д. Технология проектирования автоматизированных систем обработки информации и управления. Москва: Горячая линия-Телеком, 2011. 304 c. https:/e.lanbook.com/book/5191 (дата обращения: 28.02.2020).

8. Соколова В.В. Разработка мобильных приложений : учебное пособие. Томск: ТПУ, 2014. 176 c. https:/e.lanbook.com/book/82830 (дата обращения: 28.02.2020).

9. Тугов В.В. Проектирование автоматизированных систем управления : учебное пособие / В.В. Тугов, А.И. Сергеев, Н.С. Шаров. Санкт-Петербург: Лань, 2019. 172 c. https:/e.lanbook.com/ book/123695 (дата обращения: 28.02.2020).

10. Чикалов А.Н. Схемотехника телекоммуникационных устройств: Учебное пособие для вузов : учеб. пособие / А.Н. Чикалов, С.В. Соколов, Е.В. Титов. Москва: Горячая линия-Телеком, 2016. 322 с. https://e.lanbook.com/book/94634

11. Шаньгин В.Ф. Защита информации в компьютерных системах и сетях : учеб. пособие. Москва: ДМК Пресс, 2012. 592 c. https://e. lanbook.com/book/3032

12. Шандров Б.В. Технические средства автоматизации: учебник / Б. В. Шандров, А. Д. Чудаков. М.: ИЦ «Академия», 2007. 368 с.

13. Шишмарёв В.Ю. Основы автоматического управления: учебное пособие. М.: Академия, 2008. 352 с.

14. Khamidullin M.R., Mardanshin R.G., Prozorov A.V., Karimov R.I. The Introduction of QR-Codes in Production Processes // Journal of Environmental Treatment Techniques. 2019, Special Issue on Environment, Management and Economy. P. 1097-1100.

15. Isavnin A.G., Khamidullin M.R. Determining of total expenses for the objective of equipment replacement // Life Science Journal. 2014. №11 (6). P. 704-706. 


\section{References}

1. Akmarov P.B. Kodirovanie i zashchita informatsii [Coding and protection of information]. Izhevsk: Izhevskaya GSKhA, 2016, 136 p. https://e.lanbook.com/book/133975

2. Bogachev K.Yu. Osnovy parallel'nogo programmirovaniya [Fundamentals of Parallel Programming]. Moscow: BINOM. Laboratoriya znaniy, 2015, 345 p. https://ibooks.ru/reading.php?productid $=350082$

3. Garibov A.I. Osnovy razrabotki prilozheniy dlya mobil'nykh ustroystv na platforme Windows Phone [Windows Phone Mobile App Development Fundamentals]. Moscow: INTUIT, 2016, 459 p. https://e.lanbook. com/book/100344

4. Ivanova G.S. Tekhnologiya programmirovaniya [Programming technology]. M.: KNORUS, 2011. 336 p.

5. Podbel'skiy V.V. Yazyk deklarativnogo programmirovaniya XAML [XAML declarative programming language]. Moscow: DMK Press, 2018, 336 p. https://e.lanbook.com/book/111428

6. Ponomarenko V.I., Karavaev A.S. Ispol'zovanie platformy Arduino $\mathrm{v}$ izmereniyakh i fizicheskom eksperimente [Using the Arduino platform in measurements and physical experiment]. Izvestiya Vuzov. PND, 2014, vol. 22, no. 4, pp. 77-90.

7. Rudinskiy I.D. Tekhnologiya proektirovaniya avtomatizirovannykh sistem obrabotki informatsii $i$ upravleniya [Design technology for automated information processing and control systems]. Moscow: Goryachaya liniya-Telekom, 2011, 304 p. https:/e.lanbook.com/ book/5191

8. Sokolova V.V. Razrabotka mobil'nykh prilozheniy [Development of mobile applications]. Tomsk: TPU, 2014, 176 p. https://e.lanbook.com/ book $/ 82830$

9. Tugov V.V., Sergeev A.I., Sharov N.S. Proektirovanie avtomatizirovannykh sistem upravleniya [Design of automated control systems]. Sankt-Peterburg: Lan, 2019, 172 p. https://e.lanbook.com/book/123695

10. Chikalov A.N., Sokolov S.V., Titov E.V. Skhemotekhnika telekommunikatsionnykh ustroystv: Uchebnoe posobie dlya vuzov [Circuitry of 
telecommunication devices]. Moscow: Goryachaya liniya-Telekom, 2016, 322 p. https://e.lanbook.com/book/94634

11. Shan'gin V.F. Zashchita informatsii v komp'yuternykh sistemakh $i$ setyakh [Information protection in computer systems and networks]. Moscow: DMK Press, 2012, 592 p. https://e.lanbook.com/ book $/ 3032$

12. Shandrov B.V., Chudakov A. D. Tekhnicheskie sredstva avtomatizatsii [Technical means of automation]. M.: ITs "Akademiya", 2007, 368 p.

13. Shishmarev V.Yu. Osnovy avtomaticheskogo upravleniya [Fundamentals of automatic control]. Moscow: Akademiya, 2008, 352 p.

14. Khamidullin M.R., Mardanshin R.G., Prozorov A.V., Karimov R.I. The Introduction of QR-Codes in Production Processes. Journal of Environmental Treatment Techniques. 2019, Special Issue on Environment, Management and Economy, pp. 1097-1100.

15. Isavnin A.G., Khamidullin M.R. Determining of total expenses for the objective of equipment replacement. Life Science Journal, 2014, no. 11 (6), pp. 704-706.

\section{ДАННЫЕ ОБ АВТОРАХ}

Ахметов Линар Марселевич, студент

Набережночелнинский филиал Казанского национального исследовательского технического университета им.А.Н. Туполева

Академика Королева, 1, г. Набережные Челны, 423814, Российская Федерация

diobrandmayer@gmail.com

\section{Биков Данир Инсафович, студент}

Набережночелнинский филиал Казанского национального исследовательского технического университета им.А.Н. Туполева

Академика Королева, 1, г. Набережные Челны, 423814, Российская Федерация danir.bikov@gmail.com 
Хамидуллин Марат Раисович, доцент, кандидат экономических наук

Набережночелнинский филиал Казанского нациионального исследовательского технического университета им.А.Н. Туполева

Академика Королева, 1, г. Набережные Челны, 423814, Российская Федерация nayka_prom@mail.ru

\section{DATA ABOUT THE AUTHORS}

Linar M. Akhmetov, student

Kazan National Research Technical University, Branch in Naberezhnye Chelny

1, Akademik Korolev Str., Naberezhnye Chelny, 423814, Russian Federation

diobrandmayer@gmail.com

Danir I. Bikov, student

Kazan National Research Technical University, Branch in Naberezhnye Chelny

1, Akademik Korolev Str., Naberezhnye Chelny, 423814, Russian Federation

danir.bikov@gmail.com

Marat R. Khamidullin, PhD in Economics

Kazan National Research Technical University, Branch in Naberezhnye Chelny

1, Akademik Korolev Str., Naberezhnye Chelny, 423814, Russian Federation

nayka_prom@mail.ru

ORCID: 0000-0002-3326-0955 